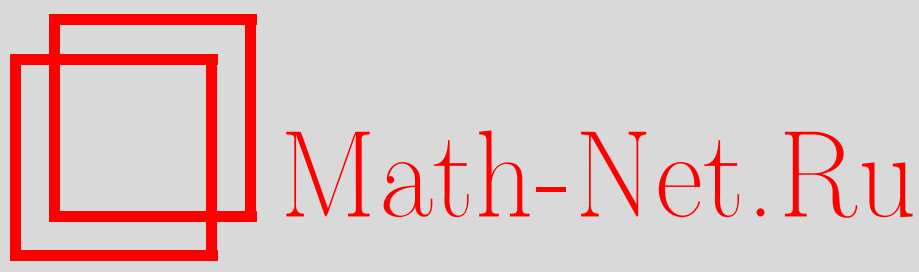

G. G. Baghaturia, M. Z. Menteshashvili, A general integral of a quasilinear equation and application to a nonlinear characteristic problem, Sibirsk. Mat. Zh., 2019, Volume 60, Number 6, 1209-1222

DOI: https://doi.org/10.33048/smzh.2019.60.602

Use of the all-Russian mathematical portal Math-Net.Ru implies that you have read and agreed to these terms of use http://www . mathnet.ru/eng/agreement

Download details:

IP: 52.6 .47 .48

April 26, 2023, 12:53:35 
Сибирский математический журнал

Ноябрь-декабрь, 2019. Том 60, № 6

УДК 517.956

\title{
ОБЩИЙ ИНТЕГРАЛ КВАЗИЛИНЕЙНОГО УРАВНЕНИЯ И ЕГО ПРИМЕНЕНИЕ ДЛЯ РЕШЕНИЯ НЕЛИНЕЙНОЙ ХАРАКТЕРИСТИЧЕСКОЙ ЗАДАЧИ
}

\section{Г. Г. Багатуриа, М. З. Ментешашвили}

\begin{abstract}
Аннотация. Описан метод построения общих интегралов для некоторых квазилинейных уравнений нестрого гиперболического типа. Доказан нелинейный аналог теоремы Асгейрссона. С помощью общего интеграла изучается нелинейный вариант характеристической задачи Гурса.
\end{abstract}

DOI 10.33048/smzh.2019.60.602

Ключевые слова: квазилинейное гиперболическое уравнение, параболическое вырождение, характеристика, общий интеграл, теорема Асгейрссона, задача Гурса.

\section{1. Введение}

Теория построения общих интегралов имеет давнюю историю, которая берет начало из фундаментальных трудов Монжа [1] и Дарбу [2,3]. Следует отметить, что построение общих решений уравнений с частными производными связано с принципиальными трудностями, но они играют значительную роль хотя бы потому, что их комбинация с другими методами упрощает исследование задач, поставленных для этих уравнений (см. [4]).

В данной работе на основании теории характеристик построены общие интегралы для некоторых нестрого гиперболических уравнений. Само построение общего интеграла уравнения не связано с какими-нибудь задачами (начальными, краевыми или другими). Структура общих решений зависит только от данного уравнения, и само решение в определенном смысле равносильно уравнению. Для решения начальных и характеристических задач в более ранних работах $[5,6]$ мы использовали представления общих интегралов для некоторых уравнений. Следует отметить работы Дж. Гвазава $[7,8]$, Р. Бицадзе [9], где для некоторых классов квазилинейных уравнений построены общие представления решений на основе метода характеристик. Мы рассматриваем новый класс уравнений, где коэффициенты при вторых производных содержат квадраты первых производных от искомого решения; оба семейства характеристик зависят от неизвестной функции. Данный класс уравнений примечателен еще тем, что он допускает параболическое вырождение, которое также зависит от неизвестного решения. Одно из уравнений этого класса допускает также вырождение порядка. Вышеизложенные свойства класса уравнений усложняют построение общего интеграла, а также постановку и исследование характеристической задачи. В данной работе удалось для конкретных уравнений этого класса построить общие интегралы в виде суммы двух произвольных функций.

(c) 2019 Багатуриа Г. Г., Ментешашвили М. 3. 
Также сформулирован аналог принципа Асгейрссона для целого класса уравнений и исследована характеристическая задача. С учетом отсутствия единой теории для нелинейных уравнений исследование отдельного класса и отдельных уравнений имеет определенное значение, так как подобными исследованиями расширяется класс квазилинейных уравнений, для которых строится общий интеграл и корректно ставятся разные задачи.

Здесь представлено систематическое изложение построения интегралов на основе метода характеристик и их применение для решения нелинейной характеристической задачи.

\section{2. Построение общих интегралов}

1. На плоскости переменных $x, y$ рассмотрим класс нестрого гиперболических квазилинейных уравнений

$$
L(u)=\Phi\left(x, y, u, u_{x}, u_{y}\right),
$$

где главная часть представляется оператором

$$
L(u) \equiv\left(u_{y}^{2}-u_{y}\right) u_{x x}-\left(2 u_{x} u_{y}+u_{y}-u_{x}-1\right) u_{x y}+\left(u_{x}^{2}+u_{x}\right) u_{y y},
$$

а правая часть $\Phi$ уравнения (1) в общем случае - заданная функция пяти переменных, определенная и непрерывно дифференцируемая на плоскости независимых переменных $(x, y)$ и определенная для всех конечных значений аргументов $u, u_{x}, u_{y}$. Характеристические корни оператора $L$

$$
\lambda_{1}=-\frac{p+1}{q}, \quad \lambda_{2}=-\frac{p}{q-1}
$$

зависят от производных $u_{x}, u_{y}$ неизвестного решения и определяют в каждой точке два характеристических направления (здесь и ниже будем использовать обозначения Монжа: $p \equiv u_{x}, q \equiv u_{y}$ ). Эти направления могут совпадать, что выражается условием

$$
p-q+1=0
$$

Для тех решений, вдоль которых выполняется условие (4), данное уравнение параболического типа. Следовательно, условием (4) определяется класс параболических решений уравнения (1). Когда для некоторого конкретного решения условие (4) всюду нарушено, вдоль него уравнение (1) гиперболического типа и условием

$$
p-q+1 \neq 0
$$

определяется класс гиперболических решений уравнения (1). В случае, когда условие (4) выполняется только в изолированных точках или на линиях, (1) является уравнением гиперболического типа с параболическим вырождением. Ввиду вышесказанного уравнение (1) принадлежит классу нестрого гиперболических уравнений.

Соотношением (1) описывается довольно широкий класс уравнений. Как известно (см. [3]), для построения общего интеграла достаточно иметь промежуточные интегралы, а в свою очередь, эти промежуточные интегралы получаются на основе первых интегралов характеристических дифференциальных соотношений и характеристических инвариантов. 
Дифференциальные соотношения (см., например, $[3,7])$, соответствующие характеристическому корню $\lambda_{1}$, имеют вид

$$
\left\{\begin{array}{l}
(p+1) d x+q d y=0, \\
d p-\frac{p}{q-1} d q-\frac{\Phi}{q^{2}-q} d x=0, \\
d u=p d x+q d y
\end{array}\right.
$$

Дифференциальные соотношения, соответствующие характеристическому корню $\lambda_{2}$, запишутся таким образом:

$$
\left\{\begin{array}{l}
p d x+(q-1) d y=0 \\
d p-\frac{p+1}{q} d q-\frac{\Phi}{q^{2}-q} d x=0 \\
d u=p d x+q d y
\end{array}\right.
$$

Особо следует отметить, что при выполнении условия (4) эти две системы характеристик сливаются и получается одна система, что всегда характерно для параболического вырождения.

Так как каждая из систем (6), (7) состоит из трех уравнений и содержит пять переменных: $x, y, u, p, q$, не следует рассматривать их как обычные системы дифференциальных уравнений, но для них можно поставить вопрос о построении первых интегралов.

Обозначим через $\xi(x, y, u, p, q)=$ const первый интеграл системы (6). Из условия $d \xi=0$ и системы (6) получаем

$$
\left\{\begin{array}{l}
M_{1}(\xi):=\xi_{x}-\frac{p+1}{q} \xi_{y}-\xi_{u}+\frac{\Phi}{q^{2}-q} \xi_{p}=0 \\
M_{2}(\xi):=p \xi_{p}+(q-1) \xi_{q}=0
\end{array}\right.
$$

Эти два уравнения линейно независимы и совместны, но они не определяют полную систему (см. [10]). Для сведения системы к полной расширим ее при помощи скобок Пуассона:

$$
M_{3}(\xi):=M_{1}\left(M_{2}(\xi)\right)-M_{2}\left(M_{1}(\xi)\right)=\xi_{y}=0 .
$$

Дальнейшее применение скобок Пуассона к расширенной системе дает новые уравнения, которые представимы линейными комбинациями входящих в нее уравнений:

$$
\left\{\begin{array}{l}
M_{4}(\xi):=M_{1}\left(M_{3}(\xi)\right)-M_{3}\left(M_{1}(\xi)\right)=0 \\
M_{5}(\xi):=M_{2}\left(M_{3}(\xi)\right)-M_{3}\left(M_{2}(\xi)\right)=0
\end{array}\right.
$$

В случае, когда однородная система, составленная из пяти уравнений (8)(10), линейно независима, она не допускает нетривиальных решений. Следовательно, первый интеграл системы (6) не может зависеть от аргументов $x, y, p$, $q, u$ и может быть только постоянным. В таких случаях при помощи характеристик построить интеграл уравнения (1) невозможно. Аналогичные рассуждения проводятся и для системы (7).

Из класса уравнений (1) рассмотрим конкретные уравнения, для которых с использованием характеристик удается построить общий интеграл.

2. Сначала рассмотрим уравнение (1) для $\Phi \equiv 0$ :

$$
\left(u_{y}^{2}-u_{y}\right) u_{x x}-\left(2 u_{x} u_{y}+u_{y}-u_{x}-1\right) u_{x y}+\left(u_{x}^{2}+u_{x}\right) u_{y y}=0 .
$$


Теорема 1. Общий интеграл уравнения (11) представляется формулой

$$
f(u+x)+g(u-y)=x,
$$

где $f, g$ - произвольные дважды непрерывно дифференцируемые функции на $R^{1}$.

ДокАЗАТЕЛЬСТво. Для уравнения (11) система соотношений

$$
\left\{\begin{array}{l}
(p+1) d x+q d y=0 \\
(1-q) d p+p d q=0
\end{array}\right.
$$

соответствует характеристическому корню $\lambda_{1}$, а другая система, определенная корнем $\lambda_{2}$, имеет вид

$$
\left\{\begin{array}{l}
p d x+(q-1) d y=0 \\
q d p-(p+1) d q=0
\end{array}\right.
$$

Как и в $(6),(7)$, к обеим системам (13) и (14) добавляется уравнение совместности

$$
d u=p d x+q d y
$$

Начнем с рассмотрения (13). Вводя обозначение $\xi(x, y, u, p, q)$ для первого интеграла системы (13), получаем систему двух линейных дифференциальных уравнений первого порядка:

$$
\left\{\begin{array}{l}
L_{1}(\xi):=\xi_{x}-\xi_{u}-\frac{p+1}{q} \xi_{y}=0, \\
L_{2}(\xi):=\xi_{p}+\frac{q-1}{p} \xi_{q}=0 .
\end{array}\right.
$$

Для сведения (16) к полной системе в смысле Якоби расширим ее при помощи скобок Пуассона

$$
L_{3}(\xi):=L_{1}\left(L_{2}(\xi)\right)-L_{2}\left(L_{1}(\xi)\right)=\frac{p-q+1}{q^{2}(1-q)} \xi_{y}=0 .
$$

Как видно, уравнение (17) линейно независимо с уравнениями системы (16). Дальнейшее применение скобок Пуассона к расширенной системе дает новые уравнения, которые представимы линейными комбинациями входящих в нее уравнений:

$$
L_{4}(\xi):=L_{1}\left(L_{3}(\xi)\right)-L_{3}\left(L_{1}(\xi)\right) \equiv 0, \quad L_{5}(\xi):=L_{2}\left(L_{3}(\xi)\right)-L_{3}\left(L_{2}(\xi)\right) \equiv 0 .
$$

Следовательно, однородная система $L_{k}(\xi)=0, k=1,2,3$, где операторы $L_{k}$ определены формулами (16), (17), полная по Якоби. Согласно теореме Якоби (см. [3]) так как система из трех уравнений полная, она допускает $n-3$ отличных друг от друга первых интегралов, где $n$ - количество переменных. В нашем случае $n=5$. Поэтому система (13), (15) имеет два, и только два, независимых дважды непрерывно дифференцируемых первых интеграла, которые легко можно получить непосредственно интегрированием уравнений системы. Интегрирование второго уравнения системы (13) дает первый интеграл

$$
\xi_{1}=\frac{p}{1-q},
$$

а второе уравнение системы (13) вместе с (15) определяют интеграл

$$
\xi=u+x .
$$


Аналогично исследуя (14), (15), заключаем, что эта система тоже имеет ровно два независимых дважды непрерывно дифференцируемых первых интеграла, которые определяются явно:

$$
\eta=u-y, \quad \eta_{1}=\frac{p+1}{q} .
$$

Выражения $\xi, \xi_{1}$ являются первыми интегралами системы (13), (15). Любая функция от этих двух величин $\xi, \xi_{1}$ также является интегралом той же системы. Аналогично рассуждая относительно системы (14), (15), можно заключить, что любая функция от двух величин $\eta, \eta_{1}$ является интегралом соответствующей системы. Отсюда следует, что между характеристическими инвариантами $\xi, \xi_{1}$ и инвариантами $\eta, \eta_{1}$ попарно существуют функциональные связи:

$$
\xi_{1}=F(\xi), \quad \eta_{1}=G(\eta)
$$

где $F, G$ - произвольные функции класса $C^{2}\left(R^{1}\right)$. Следовательно, уравнение (11) допускает ровно два промежуточных интеграла, представленные в терминах характеристических инвариантов в виде (21). В исходных переменных с учетом (18)-(20) они имеют вид

$$
\frac{p}{1-q}=F(u+x), \quad \frac{p+1}{q}=G(u-y) .
$$

Определяем из системы (22) величины $p, q$ и, подставляя соответствующие выражения в уравнение совместности (15), будем иметь

$$
d u=F \frac{G-1}{F+G} d x+\frac{F+1}{F+G} d y .
$$

Отсюда

$$
\frac{d u-d y}{G-1}+\frac{d u+d x}{F+1}=d x
$$

Введем обозначения:

$$
\frac{1}{G(u-y)-1} \equiv g^{\prime}(u-y), \quad \frac{1}{F(u+x)+1} \equiv f^{\prime}(u+x) .
$$

Подставляя их в (23), из полученного соотношения интегрированием легко приходим к (12).

Докажем, что соотношение (12) действительно является общим интегралом уравнения (11), если произвольные функции $f, g$ принадлежат классу $C^{2}\left(R^{1}\right)$. Действительно, определяем производные $f^{\prime}, g^{\prime}$ из соотношений

$$
\left\{\begin{array}{l}
\left(u_{x}+1\right) f^{\prime}(u+x)+u_{x} g^{\prime}(u-y)=0, \\
u_{y} f^{\prime}(u+x)+\left(u_{y}-1\right) g^{\prime}(u-y)=0,
\end{array}\right.
$$

полученных дифференцированием по $x, y$ равенства (12). Повторное дифференцирование соотношений (24) приводит к системе трех уравнений относительно производных $f^{\prime \prime}, g^{\prime \prime}$ :

$$
\begin{gathered}
\left(u_{x}+1\right)^{2} f^{\prime \prime}(u+x)+u_{x x} f^{\prime}(u+x)+u_{x}^{2} g^{\prime \prime}(u-y)+u_{x x} g^{\prime}(u-y)=0, \\
u_{y}^{2} f^{\prime \prime}(u+x)+u_{y y} f^{\prime}(u+x)+\left(u_{y}-1\right)^{2} g^{\prime \prime}(u-y)+u_{y y} g^{\prime}(u-y)=0, \\
\left(u_{x}+1\right) u_{y} f^{\prime \prime}(u+x)+u_{x y} f^{\prime}(u+x)+u_{x}\left(u_{y}-1\right) g^{\prime \prime}(u-y)+u_{x y} g^{\prime}(u-y)=0,
\end{gathered}
$$


где первые производные $f^{\prime}(u+x), g^{\prime}(u-y)$ уже определены из системы (24). Если из произвольных двух уравнений последней системы определить вторые производные $f^{\prime \prime}(u+x), g^{\prime \prime}(u-y)$ и внести их в оставшееся третье уравнение, то получим рассматриваемое уравнение (11). Итак, общий интеграл уравнения (11) имеет вид (12). Теорема доказана.

ЗАмЕЧАНИЕ. Легко усматривается, что общими интегралами уравнения (11) являются следующие представления:

$$
f(u+x)+g(u-y)=y,
$$

$f(u+x)+g(u-y)=u$. Нетрудно убедиться, что из-за произвольности функций $f, g$ все эти представления эквивалентны.

3. Рассмотрим уравнение, которое в правой части содержит производные первого порядка неизвестной функции $u$ :

$$
L(u)=-\frac{1}{y} p(p+1)(p-q+1) .
$$

Здесь оператор $L$ определяется по формуле (2). Сначала заметим, что правая часть этого уравнения с младшими производными перестает быть ограниченной в окрестности прямой $y=0$. В таком случае рассматриваемое уравнение можно отнести к классу нелинейных вариантов уравнения Эйлера - Дарбу (см. $[2,11,12])$. Если переписать уравнение $(26)$ в виде $y L(u)=-p(p+1)(p-q+1)$, то будет видно, что на прямой $y=0$ вырождается порядок этого уравнения. Изза наличия всех указанных особенностей в начальных и характеристических задачах выявляются эффекты слияния нелинейности, вырождения порядка и вырождения типа (см. [11]).

Теорема 2. Общий интеграл уравнения (26) представляется формулой

$$
f(u+x)+g(u-y)-y^{2}=0,
$$

где $f, g$ - произвольные дважды непрерывно дифференцируемые функции на $R^{1}$.

ДокАЗАТЕЛЬСТво. Для уравнения (26) система соотношений

$$
\left\{\begin{array}{l}
(p+1) d x+q d y=0 \\
y q(q-1) d p-y p q d q+p(p+1)(p-q+1) d x=0, \\
d u=p d x+q d y
\end{array}\right.
$$

соответствует характеристическому корню $\lambda_{1}=-\frac{p+1}{q}$, а другая система, определенная корнем $\lambda_{2}=\frac{p}{1-q}$, имеет вид

$$
\left\{\begin{array}{l}
p d x+(q-1) d y=0, \\
y q(q-1) d p-y(p+1)(q-1) d q+p(p+1)(p-q+1) d x=0, \\
d u=p d x+q d y
\end{array}\right.
$$

Для первого интеграла $\xi(x, y, u, p, q)$ системы (28), получаем два линейных дифференциальных уравнения первого порядка

$$
\left\{\begin{array}{l}
L_{1}(\xi):=\xi_{x}-\xi_{u}-\frac{p+1}{q} \xi_{y}+\frac{(p+1)(p-q+1)}{y q} \xi_{q}=0, \\
L_{2}(\xi):=\xi_{p}+\frac{q-1}{p} \xi_{q}=0
\end{array}\right.
$$


Как и в случае уравнения (11), процесс построения первых интегралов системы вида (30) сводится к интегрированию некоторой полной (в смысле Якоби) системы. Для сведения системы к полной расширим ее при помощи скобок Пуассона:

$$
L_{3}(\xi):=L_{1}\left(L_{2}(\xi)\right)-L_{2}\left(L_{1}(\xi)\right)=\frac{p-q+1}{p(p+1)^{2}}\left(\xi_{x}-\xi_{u}\right)=0 .
$$

Так как рассматриваем гиперболический случай (выполняется условие (5)), $L_{3}(\xi)$ запишется следующим образом:

$$
\xi_{x}-\xi_{u}=0 .
$$

Как видно, уравнение (31) линейно независимо с уравнениями системы (30). Дальнейшее применение скобок Пуассона к расширенной системе дает новые уравнения, которые представимы линейными комбинациями входящих в нее уравнений. Следовательно, однородная система $L_{k}(\xi)=0, k=1,2,3$, где операторы $L_{k}$ определены формулами $(30),(31)$, полная по Якоби.

Перепишем систему в эквивалентном упрощенном виде:

$$
\left\{\begin{array}{l}
X_{1}(\xi):=\xi_{x}-\xi_{u}=0, \\
X_{2}(\xi):=\xi_{p}+\frac{(q-1) y}{p(p-q+1)} \xi_{y}=0, \\
X_{3}(\xi):=\xi_{q}+\frac{y}{p-q+1} \xi_{y}=0,
\end{array}\right.
$$

и введем новую группу переменных $z_{k}, k=1,2, \ldots, 5$, следующим образом:

$$
z_{1}=x, \quad z_{2}=y, \quad z_{3}=u+x, \quad z_{4}=p, \quad z_{5}=q .
$$

В терминах переменных $z_{k}(32)$ принимает вид

$$
\left\{\begin{array}{l}
Y_{1}(\xi):=\xi_{z_{1}}=0, \\
Y_{2}(\xi):=\xi_{z_{4}}+\frac{\left(z_{5}-1\right) z_{2}}{z_{4}\left(z_{4}-z_{5}+1\right)} \xi_{z_{2}}=0, \\
Y_{3}(\xi):=\xi_{z_{5}}+\frac{z_{2}}{z_{4}-z_{5}+1} \xi_{z_{2}}=0 .
\end{array}\right.
$$

Как видно из первого уравнения системы (33), интеграл $\xi$ не зависит от аргумента $z_{1}$ и остается четверка аргументов. Второе уравнение равносильно следующей системе обыкновенных дифференциальных уравнений первого порядка:

$$
\frac{d z_{4}}{1}=\frac{z_{4}\left(z_{4}-z_{5}+1\right) d z_{2}}{\left(z_{5}-1\right) z_{2}}=\frac{d z_{3}}{0}=\frac{d z_{5}}{0}
$$

два интеграла которой $z_{3}=c$ и $z_{5}=c$ находятся непосредственно. Третий интеграл $\frac{z_{4} z_{2}}{z_{4}-z_{5}+1}=c$ можно определить из уравнения

$$
\frac{d z_{4}}{z_{4}\left(z_{4}-z_{5}+1\right)}=\frac{d z_{2}}{\left(z_{5}-1\right) z_{2}}
$$

где величина $z_{5}$ играет роль параметра.

После нового регулярного преобразования переменных:

$$
t_{1}=z_{1}, \quad t_{2}=\frac{z_{4} z_{2}}{z_{4}-z_{5}+1}, \quad t_{3}=z_{3}, \quad t_{4}=z_{4}, \quad t_{5}=z_{5},
$$

уравнения $Y_{2}=0, Y_{3}=0$ принимают вид

$$
R_{2}(\xi)=\frac{\partial \xi}{\partial t_{4}}=0, \quad R_{3}(\xi)=\frac{\partial \xi}{\partial t_{5}}=0
$$


соответственно. Таким образом, установлено, что выражение $t_{2}=\frac{z_{4} z_{2}}{z_{4}-z_{5}+1}$ является первым интегралом рассматриваемой системы и в терминах исходных переменных его можно представить в виде $\xi_{1}=\frac{p y}{p-q+1}$.

Следовательно, система имеет два, и только два, независимых дважды непрерывно дифференцируемых первых интеграла и они оба представлены в явном виде формулами

$$
\xi=u+x, \quad \xi_{1}=\frac{p y}{p-q+1} .
$$

Эти первые интегралы находятся непосредственно из системы дифференциальных характеристических соотношений. Однако мы провели полный анализ характеристической системы, чтобы убедиться, что она не имеет других первых интегралов.

Рассмотрим дифференциальные характеристические соотношения (29) второго корня $\lambda_{2}$. Для первого интеграла $\eta$ этой системы также имеем систему двух уравнений:

$$
\left\{\begin{array}{l}
L_{1}(\eta):=\frac{1-q}{p} \eta_{x}+\eta_{y}+\eta_{u}-\frac{p-q+1}{y} \eta_{q}=0 \\
L_{2}(\eta):=\eta_{p}+\frac{q}{p+1} \eta_{q}=0 .
\end{array}\right.
$$

Расширяя (35) скобками Пуассона до полной по Якоби системы и упрощая полученные уравнения, будем иметь

$$
\left\{\begin{aligned}
X_{1}(\eta) & :=\eta_{x}=0 \\
X_{3}(\eta) & :=\frac{q y}{(p+1)(p-q+1)}\left(\eta_{y}+\eta_{u}\right)+\eta_{p}=0 \\
X_{2}(\eta) & :=\frac{y}{p-q+1}\left(\eta_{y}+\eta_{u}\right)-\eta_{q}=0
\end{aligned}\right.
$$

Система (36) интегрируется аналогично системе (32). Доказывается, что она также имеет два, и только два, дважды непрерывно дифференцируемых первых интеграла:

$$
\eta=u-y, \quad \eta_{1}=\frac{p+1}{p-q+1} y
$$

Системе (28) удовлетворяет любая функция от двух переменных величин $\xi, \xi_{1}$. Аналогично общий интеграл системы (29) представляется произвольной функцией аргументов $\eta, \eta_{1}$. Отсюда следует, что между характеристическими инвариантами $\xi, \xi_{1}$ и инвариантами $\eta, \eta_{1}$ попарно существуют функциональные связи, которые запишем в виде

$$
\xi_{1}=\frac{1}{2} f^{\prime}(\xi), \quad \eta_{1}=\frac{1}{2} g^{\prime}(\eta) .
$$

Здесь $f, g$ - произвольные функции класса $C^{2}\left(R^{1}\right)$. Следовательно, уравнение (26) допускает ровно два промежуточных интеграла, представленные в терминах характеристических инвариантов в виде (38). В исходных переменных эти промежуточные интегралы имеют вид

$$
\begin{gathered}
y \frac{\partial u}{\partial x}=\frac{1}{2} f^{\prime}(u+x)\left(\frac{\partial u}{\partial x}-\frac{\partial u}{\partial y}+1\right) \\
y\left(\frac{\partial u}{\partial x}+1\right)=\frac{1}{2} g^{\prime}(u-y)\left(\frac{\partial u}{\partial x}-\frac{\partial u}{\partial y}+1\right) .
\end{gathered}
$$


Здесь также будем следовать классической схеме построения общего интеграла уравнения (26) на основании промежуточных интегралов (см., например, [11]). Из (39) и (40) определим величины

$$
p=-\frac{f^{\prime}(\xi)}{f^{\prime}(\xi)+g^{\prime}(\eta)}, \quad q=-\frac{g^{\prime}(\eta)}{f^{\prime}(\xi)+g^{\prime}(\eta)},
$$

а затем подставим их в условие согласованности (15):

$$
d u=-\frac{f^{\prime}(\xi)}{f^{\prime}(\xi)+g^{\prime}(\eta)} d x+\frac{g^{\prime}(\eta)+2 y}{f^{\prime}(\xi)+g^{\prime}(\eta)} d y
$$

Интегрируя полученное равенство, легко приходим к соотношению

$$
f(\xi)+g(\eta)=y^{2}
$$

которое с учетом первых соотношений из (34), (37) принимает вид $(27)$, т. е. равносильно представлению общего интеграла для уравнения (26).

Так же, как и для уравнения (11), можно показать, что если продифференцировать (27) два раза и исключить произвольные функции $f, g$ и их производные, то получим уравнение (26), т. е. соотношение (27) действительно является общим интегралом уравнения (26) и теорема полностью доказана.

\section{3. Некоторые свойства уравнения (1)}

Для гиперболических уравнений известна теорема Асгейрссона среднего значения (см. [13]). Например, для волнового уравнения $u_{x x}-u_{y y}=0$ это можно сформулировать следующим образом: суммы значений решения на противоположных вершинах произвольного характеристического четырехугольника равны. Уравнение (1) имеет свой собственный нелинейный аналог этого свойства. Это легко следует из инвариантов уравнения (1). Обозначим вершины произвольного характеристического криволинейного четырехугольника через $\left(x_{i}, y_{i}\right), i=1, \ldots, 4$. (Противоположные стороны этого «четырехугольника» являются отрезками одного и того же семейства характеристик.) Значения решения в точках $\left(x_{i}, y_{i}\right), i=1, \ldots, 4$, обозначим через $u_{i}, i=1, \ldots, 4$, соответственно. Характеристический четырехугольник составлен в результате пересечения двух пар характеристических линий. Одна пара состоит из двух не пересекающиеся между собой линий, которые принадлежат одному и тому же семейству характеристик корня $\lambda_{1}$; обозначим их через $\lambda_{1 a}$ и $\lambda_{1 b}$. Вторая пара состоит из двух не пересекающиеся между собой линий семейства корня $\lambda_{2}$; обозначим их через $\lambda_{2 a}$ и $\lambda_{2 b}$.

Для наглядности отметим, что в точке $\left(x_{1}, y_{1}\right)$ пересекаются линии $\lambda_{1 a}$ и $\lambda_{2 a}$, в точке $\left(x_{2}, y_{2}\right)$ - линии $\lambda_{1 a}$ и $\lambda_{2 b}$, в точке $\left(x_{3}, y_{3}\right)$ - линии $\lambda_{1 b}$ и $\lambda_{2 b}$, а в точке $\left(x_{4}, y_{4}\right)$ - линии $\lambda_{1 b}$ и $\lambda_{2 a}$. Следовательно, точки $\left(x_{1}, y_{1}\right)$ и $\left(x_{2}, y_{2}\right)$ лежат на одной и той же характеристике $\lambda_{1 a}$. Значит, в этих точках выполняется равенство $u_{1}+x_{1}=u_{2}+x_{2}$. Аналогично, так как точки $\left(x_{3}, y_{3}\right)$ и $\left(x_{4}, y_{4}\right)$ лежат на одной и той же характеристике $\lambda_{1 b}$, в этих точках выполняется равенство $u_{3}+x_{3}=u_{4}+x_{4}$. В то же время точки $\left(x_{1}, y_{1}\right)$ и $\left(x_{4}, y_{4}\right)$ лежат на одной и той же характеристике $\lambda_{2 a}$. Значит, можно написать $u_{1}-y_{1}=u_{4}-y_{4}$. Аналогично выполняется равенство $u_{2}-y_{2}=u_{3}-y_{3}$ исходя из факта, что точки $\left(x_{2}, y_{2}\right)$ и $\left(x_{3}, y_{3}\right)$ лежат на одной и той же характеристике $\lambda_{2 b}$.

Учитывая, что характеристический четырехугольник берется произвольно, из полученных соотношений, исключая $u_{1}, u_{2}, u_{3}, u_{4}$, выводим, что суммы 
абсцисс и ординат противоположных вершин произвольного характеристического четырехугольника равны: $x_{1}+x_{3}+y_{1}+y_{3}=x_{2}+x_{4}+y_{2}+y_{4}$. Именно это является нелинейным аналогом среднего значения принципа Асгейрссона. Это простое свойство, объединенное с помощью других методов, значительно упрощает исследование проблем, сформулированных для уравнений (1).

ЗАмечАниЕ. Отметим, что в конкретном случае для уравнения (11) имеет место еще более сильное свойство, а именно суммы абсцисс и ординат противоположных вершин равны:

$$
x_{1}+x_{3}=x_{2}+x_{4}, \quad y_{1}+y_{3}=y_{2}+y_{4} .
$$

\section{4. Задача Гурса для уравнения (11)}

Следует отметить, что линейные постановки характеристических задач не распространяются на случай нелинейных уравнений. Основной причиной этого является зависимость характеристических семейств от неизвестного решения. В этом случае следует установить, какими должны быть носители данных. Существуют разные варианты такого подбора. Например, можно задавать некоторые комбинации решения или его производных на неизвестных характеристиках, определение которых требуется одновременно с решением.

Рассмотрим вариант нелинейной характеристической задачи Гурса в следующей постановке.

Задача Гурса (общий случай). Пусть выходящие из общей точки $\left(x_{0}, y_{0}\right)$ строго монотонные, гладкие, разомкнутые жордановы дуги $\gamma$ и $\delta$ представлены в явном виде функциями $y=\varphi(x), y=\psi(x)$ соответственно, где $\varphi \in C^{2}\left[x_{0}, x_{1}\right]$, $\psi \in C^{2}\left[x_{0}, x_{2}\right]$. Без ограничения общности будем считать, что $x_{2}>x_{1}$. Пусть функции $\varphi, \psi$ удовлетворяют следующим условиям:

$$
\begin{cases}\varphi^{\prime}(x) \neq 0 ;-1, & x \in\left[x_{0}, x_{1}\right], \\ \psi^{\prime}(x) \neq 0 ;-1, & x \in\left[x_{0}, x_{2}\right], \\ \varphi(x) \neq \psi(x), & x \in\left(x_{0}, x_{1}\right] \\ \varphi^{\prime}(x) \neq \psi^{\prime}(x), & x \in\left(x_{0}, x_{1}\right] .\end{cases}
$$

Требуется найти решение $u(x, y)$ уравнения (11) и его область определения при известном значении $u_{0}$ в точке $\left(x_{0}, y_{0}\right)$, если дуга $\gamma$ является характеристикой семейства корня $\lambda_{1}$, а дуга $\delta$ - характеристикой семейства корня $\lambda_{2}$.

Предположим, что выше определенные функций $\varphi, \psi$ вместе с (43) удовлетворяют следующим условиям: функциональные уравнения

$$
u_{0}+x_{0}-x-\varphi(x)=z, \quad u_{0}-\psi\left(x_{0}\right)+x+\psi(x)=t
$$

однозначно разрешимы относительно $x$ на сегментах $\left[x_{0}, x_{1}\right]$ и $\left[x_{0}, x_{2}\right]$ соответственно, а их решения

$$
\begin{aligned}
& x=\tau(z), \quad z \in\left[u_{0}-\varphi\left(x_{0}\right), u_{0}+x_{0}-x_{1}-\varphi\left(x_{1}\right)\right], \\
& x=\nu(t), \quad t \in\left[u_{0}+x_{0}, u_{0}+x_{2}-\psi\left(x_{0}\right)+\psi\left(x_{2}\right)\right],
\end{aligned}
$$

дважды непрерывно дифференцируемы. Постараемся построить решение задачи Гурса и найти область определения решения.

Согласно условию задачи дуга $\gamma$ принадлежит семейству характеристик корня $\lambda_{1}$. Тем самым на этой дуге значение инварианта $u+x$ всюду постоянно. Значение этой комбинации то же, что и в точке $\left(x_{0}, y_{0}\right)$. Следовательно,

$$
\left.(u(x, \varphi(x))+x)\right|_{\gamma}=u_{0}+x_{0} .
$$


Несложно определить значения характеристического инварианта $u-y$ другого семейства на этой же дуге:

$$
\left.(u(x, \varphi(x))-y)\right|_{\gamma}=u_{0}+x_{0}-x-\varphi(x) .
$$

С учетом значений этих инвариантов из представления общего интеграла (25) получаем

$$
f\left(u_{0}+x_{0}\right)+g\left(u_{0}+x_{0}-x-\varphi(x)\right)=\varphi(x) .
$$

Аналогично, так как по условию задачи дуга $\delta$ принадлежит семейству характеристик корня $\lambda_{2}$, на этой дуге всюду значение инварианта $u-y$ постоянно. Следовательно,

$$
\left.(u(x, \psi(x))-y)\right|_{\delta}=u_{0}-y_{0} .
$$

С учетом этого можно определить значение характеристического инварианта $u+x$ первого семейства на этой же дуге:

$$
\left.(u(x, \psi(x))+x)\right|_{\delta}=u_{0}-y_{0}+x+\psi(x) .
$$

Из общего интеграла (25) имеем

$$
f\left(u_{0}-y_{0}+x+\psi(x)\right)+g\left(u_{0}-\psi\left(x_{0}\right)\right)=\psi(x) .
$$

С целью окончательного определения произвольных функций $f, g$ получаем систему из уравнений (44), (45). Введем обозначения:

$$
\begin{aligned}
& u_{0}+x_{0}-x-\varphi(x)=z, \\
& u_{0}-y_{0}+x+\psi(x)=t,
\end{aligned}
$$

и попытаемся определить величину $x$ в виде функции аргумента $z$ из функционального уравнения (46). Согласно нашим предположениям это можно сделать и (46) имеет обратную $x=\tau(z)$. Аналогично определим величину $x$ в виде функций аргумента $t$ из функционального уравнения (47): $x=\nu(t)$. Следовательно, можем написать

$$
\begin{gathered}
f\left(u_{0}+x_{0}\right)+g(z)=\varphi(\tau(z)), \quad z \in\left[u_{0}-\varphi\left(x_{0}\right), u_{0}+x_{0}-x_{1}-\varphi\left(x_{1}\right)\right], \\
f(t)+g\left(u_{0}-\psi\left(x_{0}\right)\right)=\psi(\nu(t)), \quad t \in\left[u_{0}+x_{0}, u_{0}+x_{2}-\psi\left(x_{0}\right)+\psi\left(x_{2}\right)\right] .
\end{gathered}
$$

Подставляя в (48) и (49) вместо аргументов $z$ и $t$ соответственно $u-y, u+x$ и учитывая общий интеграл уравнения, получаем интеграл задачи в виде

$$
\psi(\nu(u+x))+\varphi(\tau(u-y))-f\left(u_{0}+x_{0}\right)-g\left(u_{0}-y_{0}\right)=y .
$$

С учетом соотношения

$$
f\left(u_{0}+x_{0}\right)+g\left(u_{0}-y_{0}\right)=y_{0},
$$

которое следует из представления общего интеграла, взятого в точке $\left(u_{0}, y_{0}\right)$, приходим к окончательному результату:

$$
\psi(\nu(u+x))+\varphi(\tau(u-y))=y+y_{0} .
$$

Таким образом, решение задачи Гурса сводится к функциональному уравнению (50). Полученный интеграл (50) характеристической задачи дает возможность определения всех характеристик, выходящих из точек обеих характеристик $\varphi(x), \psi(x)$. 
Сначала рассмотрим характеристики семейства корня $\lambda_{2}$, которые выходят из точек дуги $\gamma$. Возьмем точку $(\alpha, \varphi(\alpha))$ на дуге $\gamma$ произвольно. В этой точке известны значения решения $u: u(\alpha, \varphi(\alpha))=u_{0}+x_{0}-\alpha$. Следовательно, известно и значение инварианта $u-y$ семейства характеристик корня $\lambda_{2}$. Вдоль характеристики семейства $\lambda_{2}$, выходящей из точки $(\alpha, \varphi(\alpha))$, инвариант семейства характеристик корня $\lambda_{2}$ сохраняет постоянное значение, равное значению $u_{0}+x_{0}-\varphi(\alpha)-\alpha$. Обозначим эту характеристику через $\delta(\alpha)$. Тогда

$$
\left.(u+x)\right|_{\delta(\alpha)}=u-y+y+x=u_{0}+x_{0}-\alpha-\varphi(\alpha)+y+x .
$$

Подставляя полученные значения комбинации $u+x$ и $u-y$ в (50) - интеграл задачи, получим уравнение характеристической кривой $\delta(\alpha)$ в неявном виде:

$$
\psi\left(\nu\left(u_{0}+x_{0}-\alpha-\varphi(\alpha)+x+y\right)\right)+\varphi(\alpha)=y+y_{0} .
$$

Аналогичными рассуждениями получаем уравнение характеристической кривой $\gamma(\beta)$ семейства корня $\lambda_{1}$, выходящего из точки $(\beta, \psi(\beta))$ :

$$
\psi(\beta)+\varphi\left(\tau\left(u_{0}-y_{0}+\beta+\psi(\beta)-x-y\right)\right)=y+y_{0} .
$$

ЗАмечАНИЕ. Интеграл (50) можно представить в эквивалентном виде. Для этого (46) и (47) перепишем в виде

$$
u_{0}+x_{0}-\tau(z)-\varphi(\tau(z))=z, \quad u_{0}-y_{0}+\nu(t)+\psi(\nu(t))=t .
$$

Отсюда

$$
\varphi(\tau(z))=u_{0}+x_{0}-\tau(z)-z, \quad \psi(\nu(t))=t-u_{0}+y_{0}-\nu(t) .
$$

Внесем эти выражения в (50) и после преобразований получим

$$
\tau(u-y)+\nu(u+x)=x+x_{0} .
$$

Нетрудно убедиться в том, что решение поставленной задачи Гурса, неявно определенное уравнением (51), удовлетворяет поставленным условиям задачи.

Задача Гурса для прямолинейных характеристик. Рассмотрим случай, когда $\varphi, \psi$ - линейные функции:

$$
\varphi=a x+b, \quad \psi=c x+d .
$$

Следуя (43), будем требовать выполнения условий

$$
a \neq 0 ;-1, \quad c \neq 0 ;-1, \quad a \neq \pm c .
$$

Поставим задачу: найти решение $u(x, y)$ уравнения (11) при его известном значении $u_{0}$ в точке $\left(x_{0}, y_{0}\right)=\left(\frac{d-b}{a-c}, \frac{a d-b c}{a-c}\right)$ и область его определения, если $\varphi(x), x>x_{0}$, является характеристикой семейства корня $\lambda_{1}$, а $\psi(x), x>x_{0},-$ характеристикой семейства корня $\lambda_{2}$.

Теорема 3. Пусть функции (52) удовлетворяют условиям (53). Тогда существует решение поставленной характеристической задачи для уравнения (11) в явном виде:

$$
u=\frac{(1+a) c}{a-c} x-\frac{1+c}{a-c} y+\frac{(a+c) u_{0}+b c+d}{a-c},
$$


которая определена в области, ограниченной четырьмя характеристиками: $\varphi$, $\psi$

$$
\begin{aligned}
& y=c x+b+a \alpha-c \alpha, \\
& y=a x+d-a \beta+c \beta .
\end{aligned}
$$

ДокАЗАтЕльство. Для доказательства воспользуемся интегралом задачи, который записан в виде (51). Как известно из (46), функция $\tau$ получается обращением выражения $u_{0}+x_{0}-x-\varphi(x)$. Для нашего случая имеем $u_{0}+x_{0}-$ $x-a x-b=z$, откуда

$$
x=\frac{u_{0}+x_{0}-b-z}{1+a} \equiv \tau(z)
$$

Аналогично, учитывая (47), находим

$$
\nu(t) \equiv \frac{t-d-u_{0}+y_{0}}{1+c} .
$$

Внеся полученные выражения в (51), получим интеграл задачи:

$$
\frac{u_{0}+x_{0}-b-(u-y)}{1+a}+\frac{u+x-d-u_{0}+y_{0}}{1+c}=x+x_{0},
$$

откуда находим искомую функцию в явном виде (54). Повторяя выше проведенные рассуждения для построения характеристических кривых обоих семейств в общем случае, легко можно получить уравнения этих кривых, которые в нашем случае являются прямыми (55) и (56). Как видно, характеристики одного и того же семейства ( $\varphi$ и $(55), \psi$ и $(56))$ - параллельные прямые. Отсюда заключаем, что в области определения решения задачи нет особых точек, что в общем случае не исключается. Теорема доказана.

Следствие. В случае линейных $\varphi, \psi$ вместе с (42) имеет место то же свойство, что и для волнового уравнения:

$$
u\left(x_{1}, y_{1}\right)+u\left(x_{3}, y_{3}\right)=u\left(x_{2}, y_{2}\right)+u\left(x_{4}, y_{4}\right)
$$

ПримеР 1. В качестве примера рассмотрим случай, когда в задаче Гурса

$$
\varphi(x)=x+1, x \in[0, \alpha], \quad \psi(x)=2 x+1, x \in[0, \beta] .
$$

Они выходят из общей точки $(0,1)$, где пусть задано значение решения: $u(0,1)=$ $u_{0}$.

В данном случае условия теоремы 3 выполняются и решение поставленной задачи имеет вид $u=3 y-4 x+u_{0}-3$. Областью определения решении является характеристический четырехугольник, который ограничен отрезками прямых:

$$
y=x+1, \quad y=2 x+1, \quad y=2 x-\alpha+1, \quad y=x+\beta+1 .
$$

Легко усмотреть, что в вершинах характеристического четырехугольника $A(0,1)$, $B(\alpha, \alpha+1), C(\alpha+\beta, \alpha+2 \beta+1), D(\beta, 2 \beta+1)$ выполняется аналог теоремы Асгейрссона для уравнения (11): суммы абсцисс противоположных вершин $A, C$ и $B, D$ равны; суммы ординат противоположных вершин равны, а также вместе с (42) выполняется и (57).

Прямолинейность характеристик не является необходимым условием существования регулярного решения задачи Гурса для уравнения (11). Следующий пример - подтверждение этого факта. 
ПримеР 2. Пусть функции $\varphi$ и $\psi$ имеют вид $\varphi(x)=x+2, x \in\left[2, x_{1}\right], \psi(x)=$ $x^{2}, x \in\left[2, x_{2}\right]$. Они выходят из общей точки $(2,4)$, и пусть задано значение решения: $u(2,4)=4$.

Как видно, условия (43) выполняются и решение поставленной задачи имеет вид $u=y-2 x+1+\sqrt{4 y-4 x+1}$. Что касается семейств характеристических кривых, с одной стороны, это семейство параллельных прямых, выходящих из точек параболы $y=x^{2}, x \in\left[2, x_{2}\right]$, а с другой стороны, это семейство ветвей парабол, выходящих из точек прямой $y=x+2, x \in\left[2, x_{1}\right]$. Отсюда следует, что регулярное решение задачи определяется в криволинейном четырехугольнике, ограниченном кривыми

$y=x+2, y=x^{2}, y=x+x_{2}^{2}+x_{2}+1-\sqrt{x_{2}^{2}+x_{2}}, \sqrt{17-8 x_{1}+4 x+y}=2 x-2 x_{1}+5$.

\section{ЛИТЕРАТУРА}

1. Monge G. Mémoire sur le calcul intégral des équations aux différences partielles // Mém. Acad. Sci. Paris: Imprimerie Royale. 1784. P. 118-192.

2. Darboux $G$. Leçons sur la théorie générale des surfaces et les applications geometriques du calcul infinitésimal. Paris: Gauthier-Villars et Fils, 1894.

3. Гурса Э. Курс математического анализа. М.; Л.: Гостехиздат, 1933. Т. 3.

4. Бицадзе А. В. Некоторые классы уравнений в частных производных. М.: Наука, 1981.

5. Baghaturia G. Nonlinear versions of hyperbolic problems for one quasi-linear equation of mixed type // J. Math. Sci. 2015. V. 208, N 6. P. 621-634.

6. Ментешашвили М. З. О задаче Коши с начальными данными на единичной окружности // Сообщ. АН Груз. ССР. 1993. Т. 148, № 2. С. 190-192.

7. Гвазава Дж. К. Некоторые классы гиперболических уравнений и уравнений смешанного типа. Тбилиси: Мецниереба, 1992.

8. Gvazava J. K. Nonlocal and initial problems for quasi-linear, non-strictly hyperbolic equations with general solutions represented by superposition of arbitrary functions // Georgian Math. J. 2003. V. 10. P. 687-707.

9. Бицадзе Р. Г. Общее представление решений квазилинейного уравнения в задаче нелинейной осцилляции // Сообщ. АН Груз. ССР. 1987. Т. 128, № 3. С. 493-496.

10. Гюнтер Н. М. Интегрирование уравнений первого порядка в частных производных. М.; Л.: ОНТИ Гостехиздат, 1934.

11. Goursat E. Lecons sur l'intégration des équations aux dérivées partielles du second ordre à deux variables indépendantes. Paris: Hermann, 1898. V. 2.

12. Hörmander L. Lectures on nonlinear hyperbolic differential equations. Berlin: Springer-Verl., 1997.

13. Ásgeirsson L. Über eine Mittelwertseigenschaft von Lösungen homogener linearer partieller Differentialgleichungen 2. Ordnung mit konstanten Koeffizienten // Math. Ann. 1937. V. 113, N 1. P. 321-346.

Поступила в редакиию 5 октября 2018 г.

После доработки 9 марта 2019 г.

Принята к публикачии 24 июля 2019 г.

Багатуриа Гиорги Гурамович, Ментешашвили Марина Зауревна

Грузинский технический университет,

Институт вычислительной математики им. Н. И. Мусхелишвили,

ул. Г. Перадзе, 4, Тбилиси 0159, Грузия

nogela@gmail.com, marimen1963@gmail.com 